\title{
Some Observations on Primality Testing
}

\author{
By H. C. Williams and R. Holte
}

\begin{abstract}
Let $N$ be an integer which is to be tested for primality. Previous methods of ascertaining the primality of $N$ make use of factors of $N \pm 1, N^{2} \pm N+1$, and $N^{2}+1$ in order to increase the size of any possible prime divisor of $N$ until it is impossible for $N$ to be the product of two or more primes. These methods usually work as long as $N<K^{2}$, where $K$ is $1 / 12$ of the product of the known prime power factors of $N \pm 1, N^{2} \pm N+1$, and $N^{2}+1$. In this paper a technique is described which, when used in conjunction with these methods, will often determine the primality of $N$ when $N<l K^{3}$ and $l$ is small.
\end{abstract}

1. Introduction. Let $N$ be an integer which is to be tested for primality. In Brillhart, Lehmer and Selfridge [1] and Williams and Judd [5], [6] several methods are presented for ascertaining the primality of $N$. These methods make use of the factors of $N \pm 1, N^{2}+1$, or $N^{2} \pm N+1$ in order to increase the size of the possible prime factors of $N$ until it is impossible for $N$ to be the product of two or more primes.

The combination of these various methods has proved quite successful for testing values of $N$ up to 90 or more digits; however, it sometimes occurs that a much smaller number can be very troublesome. For example, consider the 76 digit value of $N$ below:

$N=124234067210162251532295145371764077620872877495523069552841$

6715857159207729.

This number is the large pseudoprime divisor of the Lucas number $l_{476}$. Here

$$
\begin{aligned}
N-1 & =2^{4} \cdot 7^{2} \cdot 17 \cdot 773 \cdot R_{1}, \\
N+1 & =2 \cdot 3^{4} \cdot 5 \cdot 199 \cdot 2571 \cdot R_{2}, \\
N^{2}+N+1 & =73 \cdot R_{3}, \\
N^{2}+1 & =1741 \cdot R_{4}, \\
N^{2}-N+1 & =3 \cdot 13 \cdot 31 \cdot 37 \cdot R_{6},
\end{aligned}
$$

where $R_{1}, R_{2}, R_{3}, R_{4}, R_{6}$ are all composite and have no prime divisor $<5 \times 10^{7}$. With this information it is not possible to prove $N$ a prime by using only the methods referred to above.

For a given $N$ let

$$
\begin{array}{ll}
N-1=F_{1} R_{1}, & \left(\bar{F}_{1}=F_{1} / 2\right), \\
N+1=F_{2} R_{2}, & \left(\bar{F}_{2}=F_{2} / 2\right),
\end{array}
$$

Received July 7, 1977; revised November 14, 1977.

AMS (MOS) subject classifications (1970). Primary 10A25.

Copyright $\odot$ 1978, American Mathematical Society 


$$
\begin{array}{rlrl}
N^{2}+N+1 & =F_{3} R_{3}, & \left(\bar{F}_{3}=F_{3} / 3 \text { when } 3 \mid F_{3} ; \text { otherwise } \bar{F}_{3}=F_{3}\right), \\
N^{2}+1=F_{4} R_{4}, & \left(\bar{F}_{4}=F_{4} / 2\right), \\
N^{2}-N+1=F_{6} R_{6}, & \left(\bar{F}_{6}=F_{6} / 3 \text { when } 3 \mid F_{6} ; \text { otherwise } \bar{F}_{6}=F_{6}\right),
\end{array}
$$

where $F_{1}, F_{2}, F_{3}, F_{4}, F_{6}$ are completely factored and all prime divisors of any of the $R_{i}(i=1,2,3,4,6)$ must exceed the factor bound $B$. We further assume that if $p^{\alpha}$ is a prime power divisor of any of the above polynomials in $N$ and $p<B$, then $p^{\alpha}$ appears as a factor in the appropriate $F_{i}$. Put

$$
K=F_{1} \bar{F}_{2} \bar{F}_{3} \bar{F}_{4} \bar{F}_{6}
$$

and assume that $(N, 6)=1, B>3$. In this paper we present a technique which can often be used in conjunction with the tests of [1], [5] and [6] to determine whether or not $N$ is a prime when $N<l K^{3}$ and $l$ is small.

2. $N$ the Product of Three Primes. If $N$ is not too large (not over 100 digits), it is usually possible to use the methods of [6] to show that $N$ cannot be the product of three or more primes. In this section we give another method which is sometimes useful for proving that $N$ cannot be the product of three primes. We make use of the notation of [5], [6] and we assume that $N$ has satisfied the appropriate tests of [1], [5], [6]. As we make extensive use of [5] and [6] in what follows, we will indicate, when relevant, those parts of these papers which we are referencing.

Assume $N=p_{1} p_{2} p_{3}$, where $p_{1}, p_{2}, p_{3}$ are primes and $p_{1}$ is a prime of the first kind $[5$, p. 167]. We have

$$
\begin{array}{ll}
p_{1} \equiv 1 & \left(\bmod q_{1} F_{1}\right), \\
p_{1} \equiv-1 & \left(\bmod q_{2} F_{2}\right), \\
p_{1}^{2} \equiv-1 & \left(\bmod q_{4} F_{4}\right),
\end{array}
$$

where $q_{i}$ is some prime divisor of $R_{i}(i=1,2,4)$. Let $Q$ be the largest prime divisor of $\bar{F}_{4}$ when $\bar{F}_{4}>1$; then

$$
p_{1} \equiv \lambda_{1} \quad \text { or } \quad \lambda_{2} \quad(\bmod C)
$$

where $C=Q F_{1} \bar{F}_{2}, \lambda_{1} \equiv \lambda_{2} \equiv 1\left(\bmod F_{1}\right), \lambda_{1} \equiv \lambda_{2} \equiv-1\left(\bmod F_{2}\right), \lambda_{1} \equiv N(\bmod Q)$, $\lambda_{2} \equiv-N(\bmod Q)$, and $0 \leqslant \lambda_{1}, \lambda_{2}<C$. Now $p_{2}$ and $p_{3}$ must both be of the same kind [5, p. 167], and we first assume that they are of the second kind; hence, $p_{2} \equiv$ $p_{3} \equiv \pm 1\left(\bmod F_{2}\right)$. If we choose the positive sign here, we get

$$
p_{2} \equiv p_{3} \equiv 1 \quad\left(\bmod F_{1} \bar{F}_{2}\right) \quad \text { and } \quad p_{2}>B^{2} F_{1} \bar{F}_{2}, \quad p_{3}>B^{2} F_{1} \bar{F}_{2} \text {. }
$$

If we verify by trial division that $\lambda_{i}+m C \nmid N$ for $i=1,2$ and $0 \leqslant m \leqslant T$, we cannot have $T C B^{4} F_{1}^{2} \bar{F}_{2}^{2}>N$.

Now suppose $p_{2} \equiv p_{3} \equiv-1\left(\bmod F_{2}\right)$. Since $p_{2}^{2} \equiv p_{3}^{2} \equiv 1\left(\bmod F_{4}\right)$, we see that

$$
p_{2} \equiv \nu_{1}, \nu_{2} \quad(\bmod C) \text { and } p_{3} \equiv \nu_{1}, \nu_{2} \quad(\bmod C) \text {, }
$$

where $\nu_{1} \equiv \nu_{2} \equiv 1\left(\bmod F_{1}\right), \nu_{1} \equiv \nu_{2} \equiv-1\left(\bmod F_{2}\right), \nu_{1} \equiv 1(\bmod Q), \nu_{2} \equiv-1$ $(\bmod Q)$, and $0 \leqslant \nu_{1}, \nu_{2}<C$. Let $p_{i} \equiv r_{i}(\bmod C)(i=1,2,3)$, where $0<r_{i}<C$. 
There are only three possible values for $r_{1} r_{2} r_{3}$; these are $\lambda_{1} \nu_{1}^{2}, \lambda_{1} \nu_{2}^{2}, \lambda_{2} \nu_{1} \nu_{2}$. Let $V_{1}$, $V_{2}, V_{3}$ be the three possible values of $\left(N-r_{1} r_{2} r_{3}\right) / C$. Since $N$ is of the form

$$
N=\left(m_{1} C+r_{1}\right)\left(m_{2} C+r_{2}\right)\left(m_{3} C+r_{3}\right)
$$

we see that

$$
V_{i} \equiv m_{1} r_{3} r_{2}+m_{2} r_{3} r_{1}+m_{3} r_{1} r_{2}(\bmod C) .
$$

It follows that $m_{1}+m_{2}+m_{3} \geqslant \bar{V}_{i}\left(\bmod F_{1} \bar{F}_{2}\right)$, where $\bar{V}_{i} \equiv V_{i}\left(\bmod F_{1} \bar{F}_{2}\right), 0 \leqslant$ $\bar{V}_{i}<F_{1} \bar{F}_{2}$. Let $\bar{V}=\min \left(\bar{V}_{1}, \bar{V}_{2}, \bar{V}_{3}\right)$ and verify that $m C+v_{i} \nmid N$ for $i=1,2$ and all $m \leqslant T$. Since one of $m_{1}, m_{2}, m_{3}$ must exceed $\bar{V} / 3$, we see that if $T^{2} \bar{V} C^{3} / 3>N$, we have a contradiction.

When $p_{1}, p_{2}, p_{3}$ are all of the first kind,

$$
N=\left(m_{1} C+r_{1}\right)\left(m_{2} C+r_{2}\right)\left(m_{3} C+r_{3}\right),
$$

where $r_{1} r_{2} r_{3}$ can only be $\lambda_{1}^{2} \lambda_{2}$ or $\lambda_{2}^{3}$. Let $\bar{V}_{4}$ and $\bar{V}_{5}$ be the two possible values of $\left(N-r_{1} r_{2} r_{3}\right) / C$ modulo $F_{1} \bar{F}_{2}\left(0 \leqslant \bar{V}_{4}, \bar{V}_{5}<F_{1} \bar{F}_{2}\right)$. We can use the same reasoning as that above to show that $m_{1}+m_{2}+m_{3} \geqslant \bar{V}_{i}(i=4,5)$. Thus, if

$$
V=\min \left(\bar{V}_{1}, \bar{V}_{2}, \bar{V}_{3}, \bar{V}_{4}, \bar{V}_{5}\right) \quad \text { and } \quad N<\min \left(T^{2} \bar{V} C^{3} / 3, T C B^{4} F_{1}^{2} F_{2}^{2}\right),
$$

then $N$ cannot be the product of three primes.

If this method fails because one of the inequalities above is not satisfied, another method of proceeding is to find a lower bound $B_{1}$ on a prime of the first kind which divides $N$ by using the method of $[6$, p. 878] to first find all the possible positive remainders $S_{1}, S_{2}, S_{3}, \ldots, S_{k}(\bmod K)$ of a prime factor of the first kind of $N$. We then verify that for each $S_{i}, S_{i}+m K \nmid N$ for $m=0,1,2, \ldots, T$, and put $B_{1}=$ $(T+1) K$.

If $p$ is a prime divisor of $N$ of the second kind, we must have either

and $p>\sqrt{B^{5} K}$ or

$$
\begin{array}{ll}
p^{2} \equiv 1 & \left(\bmod q_{1} F_{1}\right), \\
p^{2} \equiv 1 & \left(\bmod q_{2} F_{2}\right), \\
p^{2} \equiv 1 & \left(\bmod q_{3} F_{3}\right), \\
p^{2} \equiv 1 & \left(\bmod q_{4} F_{4}\right), \\
p^{2} \equiv 1 & \left(\bmod q_{6} F_{6}\right),
\end{array}
$$

$$
\left\{\begin{aligned}
p \equiv 1 & \left(\bmod F_{1}\right) \\
p \equiv \delta & \left(\bmod F_{2}\right) \quad(|\delta|=1) \\
p^{2}+p+1 \equiv 0 & \left(\bmod F_{3}\right) \\
p^{2} \equiv 1 & \left(\bmod F_{4}\right) \\
p^{2}+\epsilon p+1 \equiv 0 & \left(\bmod F_{6}\right) \quad(|\epsilon|=1)
\end{aligned}\right.
$$

where we cannot have $\delta=\epsilon=-1$. If the system of congruences (*) has no positive solution which divides $N$ and is also less than $B_{1}$ and if $B^{5} K>B_{1}^{2}$, then $N$ cannot be the product of three primes when $B_{1}^{3}>N$. 
3. $N$ a Product of Two Primes. If $N$ has satisfied the tests of [1] , [5] and [6] and if $N$ must be the product of at most two primes, then $N=p_{1} p_{2}$, where $p_{1}$ is a prime of the first kind and $p_{2}$ is a prime of the second kind. There are six possible cases ([5, pp. 167-168], [6, pp. 877-878]). For all of these cases we have

$$
\begin{array}{llll}
p_{1} \equiv 1 & \left(\bmod q_{1} F_{1}\right), & p_{2} \equiv 1 & \left(\bmod q_{1} F_{1}\right), \\
p_{1} \equiv-1 & \left(\bmod q_{2} F_{2}\right), & p_{2} \equiv 1 & \left(\bmod q_{2} F_{2}\right) .
\end{array}
$$

For two of these cases we have

$$
\begin{array}{llll}
p_{1} \equiv \pm N & \left(\bmod q_{4} F_{4}\right), & p_{2} \equiv \pm 1 & \left(\bmod q_{4} F_{4}\right), \\
p_{1} \equiv N & \left(\bmod q_{3} F_{3}\right), & p_{2} \equiv 1 & \left(\bmod q_{3} F_{3}\right), \\
p_{1} \equiv N & \left(\bmod q_{6} F_{6}\right), & p_{2} \equiv 1 & \left(\bmod q_{6} F_{6}\right),
\end{array}
$$

where $q_{i}$ is some prime divisor of $R_{i}$. Thus, if $s_{1} \equiv s_{2} \equiv 1\left(\bmod F_{1}\right), s_{1} \equiv s_{2} \equiv-1$ $\left(\bmod F_{2}\right), s_{1} \equiv s_{2} \equiv N\left(\bmod \bar{F}_{3} \bar{F}_{6}\right), s_{1} \equiv N\left(\bmod F_{4}\right), s_{2} \equiv-N\left(\bmod F_{4}\right), 0<s_{1}$, $s_{2}<K$, and $K m+s_{1}, K m+s_{2} \nmid N$ for $m=0,1,2, \ldots, T$, then neither of these cases can hold if $T B^{4} K F_{1} \bar{F}_{2} \bar{F}_{3} \bar{F}_{6}>N$. Since, in most cases, this inequality can be satisfied when we are dealing with a value for $N$ that is not too large, we will devote the rest of this paper to a discussion of the four remaining cases.

For another pair of cases we have

$$
\begin{array}{llll}
p_{1} \equiv \pm N & \left(\bmod q_{4} F_{4}\right), & p_{2} \equiv \pm 1 & \left(\bmod q_{4} F_{4}\right), \\
p_{1} \equiv 1 & \left(\bmod q_{3} F_{3}\right), & p_{2} \equiv N & \left(\bmod q_{3} F_{3}\right), \\
p_{1} \equiv-1 & \left(\bmod q_{6} F_{6}\right), & p_{2} \equiv-N & \left(\bmod q_{6} F_{6}\right),
\end{array}
$$

and we put $E=\bar{F}_{2}$. In the last pair of cases we have

$$
\begin{array}{llll}
p_{1} \equiv \pm N & \left(\bmod q_{4} F_{4}\right), & p_{2} \equiv \pm 1 & \left(\bmod q_{4} F_{4}\right), \\
p_{1} \equiv-N-1 & \left(\bmod F_{3}\right), & p_{2} \equiv-N-1 & \left(\bmod F_{3}\right), \\
p_{1} \equiv-N+1 & \left(\bmod F_{6}\right), & p_{2} \equiv N-1 & \left(\bmod F_{6}\right),
\end{array}
$$

and we put $E=\bar{F}_{2} \bar{F}_{6}$. For all of these cases we see that

$$
p_{1} \equiv-p_{2} \quad(\bmod E)^{*}
$$

and in each of them we can find $r_{1}, r_{2}$ such that $p_{1} \equiv r_{1}, p_{2} \equiv r_{2}(\bmod K)$ and $0<$ $r_{1}, r_{2}<K$. Thus, if $N$ is composite,

$$
N=\left(m_{1} K+r_{1}\right)\left(m_{2} K+r_{2}\right)
$$

for some nonnegative integers $m_{1}$ and $m_{2}$.

We will assume that $m_{1} \geqslant m_{2}$. We have

$$
M=\left(N-r_{1} r_{2}\right) / K=m_{1} m_{2} K+r_{1} m_{2}+r_{2} m_{1} ;
$$

* An analogous theory to that given below can also be developed for $E=\bar{F}_{1}$ in the second pair of cases and $E=\bar{F}_{1} \bar{F}_{3}$ in the last pair of cases. 
hence, $r_{1} m_{2}+r_{2} m_{1} \equiv M(\bmod K)$. Since $m_{1} \geqslant m_{2}$, we get $m_{1}-m_{2}=M_{1}+s E$, where $s \geqslant 0, M_{1} \equiv r_{2}^{-1} M(\bmod E)$ and $0 \leqslant M_{1}<E$. It follows that $m_{1} m_{2}>m_{2} s E$. Since $p_{1} p_{2}>m_{1} m_{2} K^{2}$ and $N<l K^{3}$, we see that if $m_{2} s E>l K$, we have a contradiction.

We attempt to show that $m_{2} s E>l K$. This can usually be done on a fast computer as long as $l K / E<10^{20}$. We do this by first finding $G$ a factor of $K / E$ such that $(G, E)=1$ and $E G>l K / L$, where $L$ is some preselected integer such that $L^{3}>l K / E$ $>N / E K^{2}$. We use three algorithms to show that $m_{2} s E>l K$. The first algorithm determines that either $m_{2}>L$ or $N$ is composite, the second algorithm determines that $s>L$ or $N$ is composite, and the third algorithm determines that either $s$ or $m_{2} \geqslant G$ or $N$ is composite.

Once these algorithms have been employed we know that $N$ is composite or that $m_{1}$ cannot exceed or equal $m_{2}$. If the latter occurs, we interchange the values of $r_{1}$ and $r_{2}$ and use the algorithms again. This will show that $N$ is either composite or $m_{2}$ cannot exceed or equal $m_{1}$. If this latter case occurs, we see that

$$
N \neq\left(K m_{1}+r_{1}\right)\left(K m_{2}+r_{2}\right)
$$

We repeat this entire procedure for each of the four possible pairs $\left(r_{1}, r_{2}\right)$. After this has all been done, we will know whether or not $N$ is a prime.

4. Algorithm to Show that $s$ and $m_{2}$ Exceed $L$. We first verify that $K m_{2}+$ $r_{2} \nmid N$ for all $m_{2}$ such that $0 \leqslant m_{2} \leqslant[l] .^{* *}$ Then, since $m_{1} m_{2}<l K$, we have $m_{1}<$ $K$. Since

$$
m_{1} r_{2}+m_{2} r_{1} \equiv M(\bmod K)
$$

we have $m_{1}=A_{1} m_{2}+A_{2}-\nu K$, where

Put

$$
\begin{array}{ll}
A_{1} \equiv-r_{2}^{-1} r_{1} & (\bmod K)\left(0 \leqslant A_{1}<K\right), \\
A_{2} \equiv M r_{2}^{-1} & (\bmod K)\left(0 \leqslant A_{2}<K\right) .
\end{array}
$$

$$
\nu_{1}=\left[\left(A_{1}+A_{2}\right) / K\right], \quad \mu_{1}=A_{1}+A_{2}-\nu_{1} K
$$

and define

$$
\mu_{k+1}=\mu_{k}+A_{1}-\epsilon_{k} K, \quad \nu_{k+1}=\nu_{k}+\epsilon_{k}
$$

where

$$
\epsilon_{k}= \begin{cases}0 & \text { when } \mu_{k}<K-A_{1}, \\ 1 & \text { when } \mu_{k} \geqslant K-A_{1} .\end{cases}
$$

If $k=m_{2}$, then $\nu_{k}=\nu$ and $\mu_{k}=m_{1}$.

Now since $M=m_{1} m_{2} K+r_{1} m_{2}+r_{2} m_{1}$, we have

$$
\left(M-A_{2} r_{2}\right) / K=A_{1} m_{2}^{2}+m_{2}\left(A_{2}+\left(A_{1} r_{2}+r_{1}\right) / K\right)-\nu\left(m_{2} K+r_{2}\right)
$$

** We use the notation $[\alpha]$ to denote the largest integer which is less than or equal to $\alpha$. 
Let $\Pi$ be a set of about 30 small primes such that if $\pi \in \Pi$, then $\pi \nmid K$. For each of these $\pi_{i} \in \Pi$ put

$$
\begin{aligned}
a_{i} & \equiv K^{-1} A_{1} \quad\left(\bmod \pi_{i}\right), \\
b_{i} & \equiv K^{-1}\left(A_{2}+\left(A_{1} r_{2}+r_{1}\right) / K\right) \quad\left(\bmod \pi_{i}\right), \\
c_{i} & \equiv-K^{-1}\left(M-A_{2} r_{2}\right) / K \quad\left(\bmod \pi_{i}\right), \\
d_{i} & \equiv K^{-1} r_{2} \quad\left(\bmod \pi_{i}\right),
\end{aligned}
$$

where $0 \leqslant a_{i}, b_{i}, c_{i}, d_{i}<\pi_{i}$. Tabulate modulo $\pi_{i}$ the values of

$$
f_{i, k} \equiv\left(a_{i} k^{2}+b_{i} k+c_{i}\right)\left(k+d_{i}\right)^{-1}\left(\bmod \pi_{i}\right)
$$

for all values of $k\left(\bmod \pi_{i}\right)$ except $k \equiv-d_{i}\left(\bmod \pi_{i}\right)$. We note that if $m_{2} \equiv k\left(\bmod \pi_{i}\right)$, then $\nu \equiv f_{i, k}\left(\bmod \pi_{i}\right)$.

To determine that $m_{2}>L$ we simply calculate each $v_{k}$ for $k=1,2,3, \ldots, L$, and find some $\pi_{i}$ such that $\nu_{k} \not \equiv f_{i, k}\left(\bmod \pi_{i}\right)$. If, for some value of $k, v_{k} \equiv f_{i, k}$ $\left(\bmod \pi_{i}\right)$ for each $\pi_{i} \in \Pi$, trial divide $N$ by $r_{2}+k K$. If $r_{2}+k K$ divides $N, N$ is composite.

In order to show that $s>L$ we use the result

$$
m_{1}=M_{1}+m_{2}+s E
$$

together with

$$
N=K^{2} m_{1} m_{2}+K r_{2} m_{1}+K r_{1} m_{2}+r_{1} r_{2}
$$

If we put $X=K m_{2}$ and substitute for $m_{1}$ in the formula for $N$, we get

$$
N=X^{2}+X\left(K M_{1}+K E s+r_{1}+r_{2}\right)+K M_{1} r_{2}+K E r_{2} s+r_{1} r_{2} .
$$

Since $X$ is an integer, we must have

$$
\begin{aligned}
h(s) & =\left(K M_{1}+K E s+r_{1}+r_{2}\right)^{2}-4\left(K M_{1} r_{2}+K E r_{2} s+r_{1} r_{2}-N\right) \\
& =\left(K M_{1}+r_{1}-r_{2}+K E s\right)^{2}+4 N,
\end{aligned}
$$

a perfect integer square.

In order to show that $h(s)$ is not a perfect square for any nonnegative $s \leqslant L$, we select $\pi \in \Pi$ and find those values of $s(\bmod \pi)$ such that $(h(s) \mid \pi)=-1$ (Legendre symbol) and then eliminate all such $s \leqslant L$. We then take another prime from $\Pi$ and eliminate more $s$ values. We continue sieving in this way until all values of $s \leqslant L$ have been eliminated. If there are still some $s$ values left over after all the $\pi \in \Pi$ have been used, then some further primes can be used. If after this there is still a value of $s$ which is not eliminated, $h(s)$ may be a perfect square. Find $Y=\sqrt{h(s)}$. If $Y$ is an integer, then since $K M_{1}+K E s+r_{1}-r_{2}<N$, we must have $Y-K M_{1}+r_{2}-r_{1}-K E s>2$, and $N$ is composite.

5. Some Results Concerning $m_{2}$. We must now devise a technique to show that $s \geqslant G$ or $m_{2} \geqslant G$. In order to do this we require some preliminary results, which we will develop in this section. 
We first find $\lambda, \kappa$ such that $0 \leqslant \lambda, \kappa<G$ and $s \equiv \lambda m_{2}+\kappa(\bmod G)$. Since $m_{1} r_{2}+m_{2} r_{1} \equiv M(\bmod G)$ and $m_{1}=M_{1}+m_{2}+s E$, we have

$$
\begin{array}{ll}
\lambda \equiv-\left(r_{2} E\right)^{-1}\left(r_{1}+r_{2}\right) & (\bmod G), \\
\kappa \equiv\left(r_{2} E\right)^{-1}\left(M-r_{2} M_{1}\right) & (\bmod G) .
\end{array}
$$

Select a factor $H$ of $K / E G$ such that $(H, G)=1\left(H^{2} \approx G\right)$ and then determine $\alpha, \beta$ such that $0 \leqslant \alpha, \beta<H$ and $u=\alpha m_{2}+\beta(\bmod H)$, where $s=\lambda m_{2}+\kappa-u G$. By using the formulas above, we get

$$
\begin{array}{ll}
\alpha \equiv\left(r_{2} E G\right)^{-1}\left(r_{1}+r_{2}+r_{2} E \lambda\right) & (\bmod H), \\
\beta \equiv\left(r_{2} E G\right)^{-1}\left(r_{2} E \kappa+r_{2} M_{1}-M\right) & (\bmod H) .
\end{array}
$$

Our method of showing that either $s \geqslant G$ or $m_{2} \geqslant G$ consists of assuming that $s$, $m_{2}<G$ and determining that this cannot be so. Under our assumption we have $0 \leqslant$ $L<\lambda m_{2}+\kappa-G u<G$ and $u=\alpha m_{2}+\beta-v H$; consequently, $\rho m_{2}+\sigma<v<\rho m_{2}$ $+\sigma+1 / H$, where $\rho=(G \alpha-\lambda) / G H, \sigma=(G \beta-\kappa) / G H$. It follows that if $H \geqslant 2$, then $v=\left[\rho m_{2}+\sigma\right]+1$. Denoting by $\{\gamma\}$ the value of $\gamma-[\gamma]$, we must have $1>$ $\left\{\rho m_{2}+\sigma\right\}>1-1 / H$.

If $m_{2}=h+k H$, then $k \leqslant[G / H]$ and $0 \leqslant h<H$. Also, $\left\{\rho m_{2}+\sigma\right\}=\{\{p h+\sigma\}$ $+1-\{\lambda k / G\}\}$. We now have two cases.

Case 1. $\{\rho k+\sigma\} \geqslant\{\lambda k / G\}$. In this case we have

$$
\left\{\rho m_{2}+\sigma\right\}=\{\rho h+\sigma\}-\{\lambda k / G\} .
$$

If $\{\rho h+\sigma\} \leqslant 1-1 / H$, then so is $\left\{\rho m_{2}+\sigma\right\}$. If $\{\rho h+\sigma\}>1-1 / H$ and $\left\{\rho m_{2}+\sigma\right\}$ $>1-1 / H$, we must have $\{k \lambda / G\}<1 / H$. If we find all pairs $(h, k)$ such that $0 \leqslant$ $h<H,\{\rho h+\sigma\}>1-1 / H, 0 \leqslant k \leqslant[G / H]$, and $\{k \lambda / G\}<1 / H$ and verify for each such pair that $K m_{2}+r_{2} \nmid N$ for $m_{2}=h+k H$, we will see that Case 1 cannot occur.

Case 2. $\{\rho h+\sigma\}<\{\lambda k / \sigma\}$. Here we have

$$
\left\{\rho m_{2}+\sigma\right\}=\{\rho h+\sigma\}-\{k \lambda / G\}+1
$$

thus, if $\left\{\rho m_{2}+\sigma\right\}>1-1 / H$, we find that

$$
\{\rho h+\sigma\}<\{k \lambda / G\}<\{\rho h+\sigma\}+1 / H .
$$

If we find all pairs $(h, k)$ such that this is so and verify that $K m_{2}+r_{2}+N$ for $m_{2}=$ $h+H k$, we will see that Case 2 cannot occur.

In order to eliminate Case 1 or Case 2 , we must begin by sorting the lists $\{\rho h+\sigma\}$, $h=0,1,2,3, \ldots, H-1$, and $\{k \lambda / G\}, k=0,1,2,3, \ldots,[G / H]$. We make use of the following theorem (see, for example, Slater [3]).

THEOREM. If the list $\{k \theta\}$, where $0<\theta<1, k=1,2,3, \ldots, n$, is sorted in ascending order, the interval $[0,1]$ is partitioned into only three distinct lengths. These are given by $x=\{a \theta\}, y=1-\{b \theta\}, z=x+y$, where $\{a \theta\}$ is the minimum element of the list and $\{b \theta\}$ is the maximum.

We call the integers $a,-b, a-b$ the integers corresponding to $x, y, z$, respectively. In [3] a fast and simple algorithm, which uses continued fractions, is given for calculating $a$ and $b$ when $\theta$ is rational or irrational. 
6. An Algorithm to Show that $m_{2}$ or $s \geqslant G$. Find, by searching the list $\{\rho h+\sigma\}$, $h=0,1,2, \ldots, H-1$, that integer $\eta$ such that $\{\rho \eta+\sigma\}$ is the least element of the list. Let $P$ be the positive remainder on dividing $(G \alpha-\lambda) \eta+G \beta-\kappa$ by $G H$ : then $\{\rho \eta+\sigma\}=P / G H$. Let the three lengths for $\theta=\rho, n=H$ be $x_{1}, y_{1}, z_{1}$ with corresponding integers $a_{1},-b_{1}, a_{1}-b_{1}$. If $t$ is any of these lengths, we can write it as $\Gamma / G H$ for an integer $\Gamma$; for, if $\gamma$ is the integer corresponding to $t, \Gamma$ is the positive remainder on dividing $\gamma(G \alpha-\lambda)$ by $G H$ when $\gamma>0$ and $\Gamma$ is $G H$ decreased by the remainder on dividing $|\gamma|(G \alpha-\lambda)$ by $G H$ when $\gamma<0$. Arrange the three possible $\Gamma$ 's into ascending order $\Gamma_{1}, \Gamma_{2}, \Gamma_{3}$ with corresponding integers $\gamma_{1}, \gamma_{2}, \gamma_{3}$.

Let the lengths for $\theta=\lambda / G, n=[G / H]$ be $x_{2}, y_{2}, z_{2}$ with corresponding integers $a_{2},-b_{2}, a_{2}-b_{2}$. As before, we can represent any length $t$ as $\Delta / G$, where $\Delta$ is an integer. Arrange the possible $\Delta$ 's into ascending order $\Delta_{1}, \Delta_{2}, \Delta_{3}$ with corresponding integers $\delta_{1}, \delta_{2}, \delta_{3}$.

Let

$$
\begin{array}{cl}
\{\rho h+\sigma\}=C_{h} / G H & \left(0<C_{h}<G H\right), \\
\{k \lambda / G\}=D_{k} / G H & \left(0<D_{k}<G H\right) .
\end{array}
$$

For Case 1 we wish $(h, k)$ such that $C_{h} / G H>1-1 / H$ and $D_{k} / G H<1 / H$, i.e. $C_{h}>$ $G H-G, D_{k}<G$. In Case 2 we wish $(h, k)$ such that $C_{h}<D_{k}<G+C_{h}$.

We put $h_{0}=\eta, C_{h_{0}}=P$ and define

$$
h_{i+1}=h_{i}+\gamma_{l}, \quad C_{h_{i+1}}=C_{h_{i}}+\Gamma_{l},
$$

where $l$ is the least of $1,2,3$ such that $0<h_{i+1}<H$. We also put $k_{0}=a_{2}, D_{k_{0}}=$ $D H$ and define

$$
k_{j+1}=k_{j}+\delta_{l}, \quad D_{k_{j+1}}=D_{k_{j}}+H \Delta_{l},
$$

where $l$ is the least of $1,2,3$ such that $0<k_{j+1} \leqslant[G / H]$ and $D$ is the remainder on dividing $a_{2} \lambda$ by $G$. We see that the list

$$
C_{h_{i}} / G H, \quad i=0,1,2, \ldots, H-1,
$$

is the same as the list $\{\rho h+\sigma\}, h=0,1,2, \ldots, H-1$, arranged in ascending order; also, the list

$$
D_{k_{i}} / G H, \quad i=0,1,2, \ldots,[G / H]-1,
$$

is the list $\{k \lambda / G\}, k=1,2,3, \ldots,[G / H]$, arranged in ascending order.

Our algorithm is now easy to establish. Put $i=0$ and find all $D_{k_{j}}$ such that

$$
C_{h_{i}}<D_{k_{j}}<G+C_{h_{i}} .
$$

At the same time save all $k_{t}$ such that $D_{k_{t}}<G$ (include also 0 as a value for $k_{t}$ ). Store all the $D_{k_{j}}$ and $k_{j}$ values and verify that $K\left(h_{i}+H k_{j}\right)+r_{2} \nmid N$ (we will give in the next section a fast method for doing this). Next increase $i$ by 1 and from among the stored $D_{k_{j}}$ and newly created ones store only those $D_{k_{j}}$ and $k_{j}$ which satisfy (*) and verify that $K\left(h_{i}+H k_{j}\right)+r_{2} \nmid N$ for each pair $\left(h_{i}, k_{j}\right)$. Continue this process until 
$i>H-1$. Store as well all $h_{q}$ such that $C_{h_{q}}>G H-G$. Finally, determine for each pair $\left(h_{q}, k_{t}\right)$ that $K\left(h_{q}+H k_{t}\right)+r_{2} \nmid N$ by trial division. This is not very time consuming as there are usually only a few of these pairs.

7. A Method for Verifying that $K\left(h_{i}+H k_{j}\right)+r_{2} \nmid N$. We give here a method which has proved to be very effective for determining that $K\left(h_{i}+H k_{j}\right)+r_{2} \nmid N$.

If $m_{2}=h_{i}+H k_{j}$ and $\left(m_{2} K+r_{2}\right)\left(m_{1} K+r_{1}\right)=N$, then, since $m_{1}=M_{1}+$ $m_{2}+s E, s=\lambda m_{2}+\kappa-u G$, and $u=\alpha m_{2}+\beta-v H$, we find

$$
\begin{aligned}
M-r_{2} M_{1}-r_{2} E(\kappa-\beta G)= & m_{2}\left(r_{1}+K M_{1}+K E(\kappa-\beta G)+r_{2} E(\lambda-G \alpha)+r_{2}\right) \\
& +m_{2}^{2} K(E(\lambda-G \alpha)+1)+w m_{2} G E K+w r_{2} G E,
\end{aligned}
$$

where $w=v H$. It follows that

$$
\begin{aligned}
w & =((G \alpha-\lambda) / G) m_{2}-\frac{m_{2}}{G E}+\frac{\beta G-\kappa}{G}-\frac{r_{1}+K M_{1}}{G E K}+\frac{M / G E K+r_{1} r_{2} / K^{2} G E}{m_{2}+r_{2} / K} \\
& =H\left(\rho m_{2}+\sigma\right)-\frac{m_{2}}{G E}-\frac{r_{1}+K M}{G E K}+\frac{M / G E K+r_{1} r_{2} / K^{2} G E}{m_{2}+r_{2} / K} .
\end{aligned}
$$

Since $v=\left[\rho m_{2}+\sigma\right]+1$ and $\left\{\rho m_{2}+\sigma\right\}=\{\rho h+\sigma\}-\{\lambda k / \sigma\}+1$, we see that

$$
w-H\left(\rho m_{2}+\sigma\right)=H(\{\lambda k / G\}-\{\rho h+\sigma\})=D_{k_{j}} / G-C_{h_{i}} / G
$$

Thus,

$$
\begin{aligned}
D_{k_{j}}-C_{h_{i}} & =-\frac{m_{2}}{E}-\frac{r_{1}+K M_{1}}{E K}+\frac{M / E K+r_{1} r_{2} / K^{2} E}{m_{2}+r_{2} / K} \\
& =\left(\frac{M / E K}{m_{2}+r_{2} / K}-\frac{m_{2}}{E}\right)-\frac{r_{1}}{E K}-\frac{M_{1}}{E}+\frac{r_{1} r_{2}}{K^{2} E\left(m_{2}+r_{2} / K\right)} .
\end{aligned}
$$

Now

$$
0<M_{1} / E \leqslant 1-\frac{1}{E}, \quad 0<\frac{r_{1}}{E K}<\frac{1}{E}, \quad 0<\frac{r_{1} r_{2}}{K^{2}} \frac{1}{E\left(m_{2}+r_{2} / K\right)}<\frac{r_{1}}{E K} ;
$$

hence,

$$
0<\frac{M_{1}}{E}+\frac{r_{1}}{E K}-\frac{r_{1} r_{2}}{K^{2} E\left(m_{2}+r_{2} / K\right)}<1
$$

We must have

$$
D_{k_{j}}-C_{h_{i}}=\left[\frac{M / E K}{m_{2}+r_{2} / K}-\frac{m_{2}}{E}\right],
$$

when $m_{2}=h_{i}+k_{j} H$ and $K m_{2}+r_{2} \mid N$. To determine that $K m_{2}+r_{2} \nmid N$, compare $D_{k_{j}}-C_{h_{i}}$ to the computed value of $\left[(M / E K) /\left(m_{2}+r_{2} / K\right)-m_{2} / E\right]$; only when they are a distance of 1 or less from each other do we need trial divide $N$ by $K m_{2}+r_{2}$.

The advantage of this method is that $M / E K$ is usually small enough to be stored on the computer as a double precision (or extended precision) floating point constant. Hence, these operations can be done using double (extended) precision arithmetic rather than multi-precise arithmetic. 
When it is more desirable to use integer arithmetic, we note that

$$
\frac{M / E K}{m_{2}+r_{2} / K}=\frac{M / E K}{m_{2}}\left(1+r_{2} / K m_{2}\right)^{-1}=\frac{M / E K}{m_{2}}\left(1-\frac{r_{2}}{K m_{2}}\right)+\eta,
$$

where $0<\eta<M r_{2}^{2} / E K^{3} m_{2}^{3}$. Since $m_{2}>L$ and $L^{3}>M / E K$, we see that $0<\eta<1$. Thus,

$$
\begin{array}{r}
\frac{M / E K}{m_{2}+r_{2} / K}-\frac{m_{2}}{E}-\frac{r_{1}}{E K}-\frac{M_{1}}{E}+\frac{r_{1} r_{2}}{K^{2} E\left(m_{2}+r_{2} / K\right)} \\
=\left[\frac{[M / E K]}{m_{2}}\right]-\left[\frac{\left[M r_{2} / E K^{2}\right]}{m_{2}^{2}}\right]-\left[\frac{m_{2}}{E}\right]+I,
\end{array}
$$

where $|I| \leqslant 2$. We trial divide by $K m_{2}+r_{2}$ only when $D_{k_{i}}-C_{h_{i}}$ is within 3 of

$$
\left[\frac{[M / E K]}{m_{2}}\right]-\left[\frac{\left[M r_{2} / E K^{2}\right]}{m_{2}^{2}}\right]-\left[\frac{m_{2}}{E}\right] \text {. }
$$

8. Some Examples. The above algorithms were implemented on an IBM/370-168 computer and run on 28 numbers supplied to the authors by John Brillhart. These numbers are pseudoprime divisors of various Lucas $\left(l_{n}\right)$ and Fibonacci numbers $\left(f_{n}\right)$. We give below some of the calculations performed on the number $N$ given in Section 1 .

Consider the case with $N$ assumed to be $p_{1} p_{2}$ with

$$
\begin{aligned}
& p_{1} \equiv 1\left(\bmod F_{1}\right), \quad p_{2} \equiv 1\left(\bmod F_{1}\right) \text {, } \\
& p_{1} \equiv-1 \quad\left(\bmod F_{2}\right), \quad p_{2} \equiv 1 \quad\left(\bmod F_{2}\right) \text {, } \\
& p_{1} \equiv N\left(\bmod F_{4}\right), \quad p_{2} \equiv 1\left(\bmod F_{4}\right) \text {, } \\
& p_{1} \equiv 1\left(\bmod F_{3}\right), \quad p_{2} \equiv N\left(\bmod F_{3}\right), \\
& p_{1} \equiv-1 \quad\left(\bmod F_{6}\right), \quad p_{2} \equiv-N\left(\bmod F_{6}\right),
\end{aligned}
$$

$p_{1}=m_{1} K+r_{1}, p_{2}=m_{2} K+r_{2}, m_{1} \geqslant m_{2}$. Here $E=287804745$, and we selected $L=1900000, l=8, G=77022424976, H=253487$. With these values we found

$$
\begin{aligned}
& \lambda=17422150686, \\
& \kappa=45747446332, \\
& \alpha=86832, \\
& \beta=50918, \\
& \eta=226218, \\
& P=526817664872, \\
& \Gamma_{1}=6149532242, \quad \Gamma_{2}=589944841356, \quad \Gamma_{3}=596094373598, \\
& \gamma_{1}=-30527, \quad \gamma_{2}=246350, \gamma_{3}=215823, \\
& \Delta_{1}=21636, \quad \Delta_{2}=300182, \quad \Delta_{3}=371818, \\
& \delta_{1}=-209354, \quad \delta_{2}=171493, \quad \delta_{3}=-37861 .
\end{aligned}
$$


The computer was then able to verify in less than one minute that $m_{1}$ could not exceed or equal $m_{2}$. The other cases were also run and the number was found to be prime in about 35 minutes C.P.U. time.

Of the remaining 27 numbers the following 26 were proved prime. These are the large pseudoprime divisors of $f_{395}(60), f_{401}(77), f_{447}(58), f_{463}(86), f_{473}(88)$, $f_{475}(62), f_{481}(72), f_{487}(87), f_{499}(89), l_{387}(50), l_{388}(65), l_{392}(55), l_{401}(71)$, $l_{403}(68), l_{407}(54), l_{416}(69), l_{417}(55), l_{436}(81), l_{443}(85), l_{446}(78), l_{453}(50), l_{458}(96)$, $l_{486}(68), l_{487}(99), l_{490}(56), l_{494}(79)$. The numbers in the parentheses give the number of digits in the pseudoprime. Most of these were proved prime by using only the methods of [1], [5], and [6]; however, for the occasional number on which these methods did not suffice, the algorithms described above were used successfully.

Consider now the 121 digit number $N=2^{400}-593$

$=25822498780869085896559191720030118743297057928292235128306593$

56540647622016841194629645353280137831435903171972747492783.

With $B=1.5 \times 10^{8}$ we have

$$
\begin{aligned}
& F_{1}=2 \cdot 1384711, \\
& F_{2}=2^{4} \cdot 3^{2} \cdot 3023 \cdot 23251093, \\
& F_{3}=7 \cdot 2521 \cdot 2213647 \cdot 70792627, \\
& F_{4}=2 \cdot 5 \cdot 13 \cdot 298013, \\
& F_{6}=3 \cdot 19 \cdot 43 .
\end{aligned}
$$

In spite of the size of this number, the methods of [1], [5], [6] together with those given above suffice to demonstrate that $N$ is a prime.

For the 65 digit pseudoprime divisor of $l_{470}$ $N=19809950476703891759635852223863606381827838846342829232189869441$, we have with $B=1.3 \times 10^{9}$

$$
\begin{aligned}
& F_{1}=2^{7} \cdot 5 \cdot 19 \cdot 37 \cdot 47 \cdot 139 \\
& F_{2}=2 \cdot 3^{3} \cdot 7, \\
& F_{3}=13 \cdot 31 \cdot 73 \cdot 79 \\
& F_{4}=2 \\
& F_{6}=3 .
\end{aligned}
$$

For this number $l>N / K^{3} \approx 9.2 \times 10^{9}$ is quite large. By using the methods described above it can be shown that $N$ is either prime or at most the product of two primes $p_{1}$ and $p_{2}$ and that we have either

$$
\begin{array}{llll}
p_{1} \equiv 1 & \left(\bmod q_{1} F_{1}\right), & p_{2} \equiv 1 & \left(\bmod q_{1} F_{1}\right), \\
p_{1} \equiv 1 & \left(\bmod q_{3} F_{3}\right), & p_{2} \equiv 1 & \left(\bmod q_{2} F_{2}\right),
\end{array}
$$




$$
\begin{array}{llll}
p_{1} \equiv 1 & \left(\bmod F_{1}\right), & p_{2} \equiv 1 & \left(\bmod F_{1}\right), \\
p_{1} \equiv-1 & \left(\bmod F_{2}\right), & p_{2} \equiv 1 & \left(\bmod F_{2}\right), \\
p_{1} \equiv-N-1 & \left(\bmod F_{3}\right), & p_{2} \equiv-N-1 & \left(\bmod F_{3}\right) .
\end{array}
$$

In the first case we see that

$$
p_{1}=1+m_{1} q_{1} q_{3} F_{1} \bar{F}_{3}, \quad p_{2}=1+m_{2} q_{1} q_{2} F_{1} \bar{F}_{2},
$$

and one of $m_{1}$ or $m_{2}$ is even. For if $m_{1}$ and $m_{2}$ were both odd, then $2^{8} \mid N-1$, which, since $2^{7} \| N-1$, is impossible. ${ }^{* * *}$ Thus

$$
N=p_{1} p_{2}>2 B^{4} F_{1}^{2} \bar{F}_{2} \bar{F}_{3}>N
$$

which is also not possible.

The second case is more difficult. We first find $r_{1}$ and $r_{2}$ such that $p_{1}=r_{1}+$ $m_{1} K$ and $p_{2}=r_{2}+m_{2} K$ and put $E=F_{1} \bar{F}_{3}$. Then

$$
m_{1}+m_{2}=M_{1}+s E
$$

where $M_{1} \equiv r_{1}^{-1} M(\bmod E), 0<M_{1}<E$, and $M=\left(N-r_{1} r_{2}\right) / K$. Let $L=1.9 \times 10^{6}$ and verify that $h^{\prime}(s)=\left(K M_{1}+r_{1}+r_{2}+K E s\right)^{2}-4 N$ is not a perfect square for all nonnegative $s \leqslant L$. If $m_{1}>m_{2}$, then $m_{1}>1 / 2 L E$ and $p_{2}>B^{2} F_{1} \bar{F}_{2}$; consequently,

$$
p_{1} p_{2}>(1 / 2 L E K) B^{2} F_{1} \bar{F}_{2}>N \text {. }
$$

If we assume that $m_{2}>m_{1}$, the size of $l$ in this case does not permit us to use the algorithm of Section 4 to show that $m_{1}>L$. However, by using the result that $m_{2}=A_{1}^{\prime} m_{1}+A_{2}^{\prime}-\nu K$, where

$$
A_{1}^{\prime} \equiv-r_{1}^{-1} r_{2}, \quad A_{2}^{\prime} \equiv r_{1}^{-1} M \quad(\bmod K)\left(0<A_{1}^{\prime}, A_{2}^{\prime}<K\right),
$$

together with $M=K m_{1} m_{2}+r_{1} m_{2}+r_{2} m_{1}$, we find that

$$
\nu=\left(A_{1}^{\prime} / K\right) m_{1}+A_{2}^{\prime} / K-\frac{M / K}{K m_{1}+r_{1}}-\frac{r_{1} r_{2}}{K^{2}\left(K m_{1}+r_{1}\right)}+\frac{r_{2}}{K^{2}} \text {. }
$$

Since $\nu$ is an integer,

$$
\nu=\left[\left(A_{1}^{\prime} / K\right) m_{1}+A_{2}^{\prime} / K-\frac{M / K^{2}}{m_{1}+r_{1} / K}\right]+1 .
$$

This gives us another method of calculating the value of $\nu_{n}$ in Section 4 . We first compute the value $I_{n}$ of

$$
\left[\left(A_{1}^{\prime} / K\right) n+A_{2}^{\prime} / K-\frac{M / K^{2}}{n+r_{1} / K}\right]
$$

then $\nu_{n}=I_{n}+1$. We can eliminate each possible value of $\nu_{n}$ for $n=0,1,2,3, \ldots, L$, by using the second part of the algorithm of Section 4 .

Another method of proceeding is to first trial divide $N$ by $K m_{1}+r_{1}$ for $m_{1}=$ $0,1,2, \ldots, L^{\prime}$. If $L^{\prime}<m_{1} \leqslant L$, we have 


$$
\frac{A_{1}^{\prime}\left(L^{\prime}+1\right)}{K}-\frac{M / K^{2}}{L^{\prime}+1}<\nu<\frac{A_{1}^{\prime} L}{K}-\frac{M / K^{2}}{L+1}+1 .
$$

Since $m_{2}=A_{1}^{\prime} m_{1}+A_{2}^{\prime}-\nu K$ and $N=\left(K m_{1}+r_{1}\right)\left(K m_{2}+r_{2}\right)$, we can substitute for $m_{2}$ as was done in the development of the second algorithm in Section 4 to find that the expression $\left(A_{1}^{\prime} r_{2}-r_{1}-K A_{2}^{\prime}+K^{2} \nu\right)^{2}+4 A_{1}^{\prime} N$ must be a perfect square. We can easily eliminate all the possible values of $\nu$ between the bounds above by using the sieve method described previously.

These tests were implemented and it was found that

$$
p_{1} p_{2}>K^{2} m_{1} m_{2}>1 / 2 L^{2} K^{2} E>N
$$

hence, $N$ is a prime.

9. Conclusion. On comparing the above methods to those given in [1] or previously, it is evident that the stress here has moved from positive tests for primality to more negative processes such as searching, sieving, and trial division. However, the greatly increased power of these methods to some extent makes up for this somewhat undesirable shift in emphasis. For example, consider the large prime divisor $N$ of $l_{470}$. At the authors' request D. H. Lehmer very kindly consented to use the ILLIAC IV in an attempt to find more factors of $N \pm 1, N^{2}+1, N^{2} \pm N+1$ than those given in Section 8. After using 4.75 hours of C.P.U. time, no additional factor was found with $B$ increased to 38269275600 . The techniques mentioned in this paper seem currently to be the only way of dealing with such stubborn numbers.

Probabilistic techniques such as those of Solovay and Strassen [4] run very quickly and have none of the negative aspects mentioned above; however, such methods do not prove primality but only support the likelihood of primality. Perhaps the best hope lies with ideas advanced by Miller [2]. When one of the algorithms discussed in [2] is combined with an as yet unpublished result of P. Weinberger, a fairly good test for primality can be obtained. Unfortunately, the proof of this algorithm requires the unproved Extended Riemann Hypothesis.

In any case it appears to the authors that the techniques of the present work have been pushed about as far as possible and any further advance in the problem of primality testing will probably have to be made in an entirely different direction.

Department of Computer Science
University of Manitoba
Winnipeg, Manitoba R3T 2N2, Canada

1. JOHN BRILLHART, D. H. LEHMER \& J. L. SELFRIDGE, "New primality criteria and factorizations of $2^{m} \pm 1$," Math. Comp., v. 29, 1975, pp. 620-647.

2. GARY L. MILLER, "Riemann's hypothesis and tests for primality," J. Comput. System Sci., v. 13, 1976, pp. 300-317.

3. NOEL B. SLATER, "Gaps and steps for the sequence $n \theta \bmod 1$," Proc. Cambridge Philos. Soc., v. 63, 1967, pp. $1115-1123$.

4. R. SOLOVAY \& V. STRASSEN, "A fast Monte-Carlo test for primality," SIAM J. Comput., v. 6, 1977, pp. 84-85.

5. H. C. WILLIAMS \& J. S. JUDD, "Determination of the primality of $N$ by using factors of $N^{2} \pm 1$," Math. Comp., v. 30, 1976, pp. 157-172.

6. H. C. WILLIAMS \& J. S. JUDD, "Some algorithms for prime testing using generalized Lehmer functions," Math. Comp., v. 30, 1976, pp. 867-886. 